\title{
Simultaneous solar laser emissions from three Nd:YAG rods within a single pump cavity
}

\author{
Dawei Liang $^{\mathrm{a}}$, Joana Almeida ${ }^{\mathrm{a}}$, Dário Garcia ${ }^{\mathrm{a}}$, Bruno D. Tibúrcio ${ }^{\mathrm{a}}$, Emmanuel Guillot ${ }^{\mathrm{b}}$, Cláudia R. Vistas ${ }^{\mathrm{a}}$ \\ a CEFITEC, Departamento de Física, FCT, Universidade NOVA de Lisboa, 2829-516 Campus de Caparica, Portugal \\ b PROMES-CNRS, 7 rue du Four Solaire, 66120, Font Romeu, Odeillo, France
}

\section{A R T I C L E I N F O}

\section{Keywords}

Solar-pumped laser

Laser emission

Multi-rod

Parabolic mirror

Slope efficiency, Nd:YAG

\begin{abstract}
A B S T R A C T
We report here, to the best of our knowledge, the first simultaneous emission of three continuous-wave solar laser beams by end-side-pumping three $3.0 \mathrm{~mm}$ diameter, $25 \mathrm{~mm}$ length Nd:YAG single-crystal rods within a single conical pump cavity. An aspheric fused silica lens was used to couple the concentrated solar radiation from the focal zone of a parabolic mirror with $1.0 \mathrm{~m}^{2}$ effective collection area into the laser rods within the pump cavity. $18.3 \mathrm{~W}$ multimode solar laser power was measured, resulting in 5.1\% laser slope efficiency. $0.036 \mathrm{~W}$ laser beam brightness figure of merit was also achieved for each beam, being 9 times more than that of the most efficient solar laser with $32.5 \mathrm{~W} / \mathrm{m}^{2}$ collection efficiency. Since the three laser rods were associated with their own $1064 \mathrm{~nm}$ output mirrors, it was possible to adjust individually both solar laser output power levels and beam qualities of the three laser beams by adopting different resonant cavity lengths, enabling potential solar laser applications.
\end{abstract}

\section{Introduction}

Solar-pumped laser is considered as one of the most promising technologies for solar energy research in the 21th century. The direct excitation of renewable lasers by natural sunlight may provide cost-effective solutions to free coherent optical radiation generation, leading to numerous benefits in the years to come. Solar-pumped lasers are usually considered for applications where sunlight is abundant and other forms of energy sources are scarce, especially in space (Vasile and Maddock, 2012; Guan et al., 2017). Powered by free solar energy, solar laser has also large potential for terrestrial applications such as high-temperature materials processing (Yabe et al., 2007).

Since 1966, primary parabolic mirrors have been utilized by Young (Young, 1966) and other researchers (Weksler and Shwartz, 1988; Arashi et al., 1984; Benmair et al., 1990; Lando et al., 2003; Almeida et al., 2013) to achieve tight focusing of incoming solar radiation for the excitation of a laser medium. With secondary and tertiary concentrators, solar laser collection efficiency was gradually boosted to $6.7 \mathrm{~W} / \mathrm{m}^{2}$ in 2003 (Lando et al., 2003). Significant progresses in solar laser efficiency have been achieved by Fresnel lenses solar laser pumping approaches (Yabe et al., 2007). $18.7 \mathrm{~W} / \mathrm{m}^{2}$ solar laser collection efficiency, defined as laser output power versus solar collector area, was firstly reported in 2007 by pumping a large Cr:Nd:YAG ceramic laser rod with a $1.4 \mathrm{~m}^{2}$ area Fresnel lens (Yabe et al., 2007). $19.3 \mathrm{~W} / \mathrm{m}^{2}$ laser collection efficiency was later achieved in 2011 by exciting a $4 \mathrm{~mm}$ diameter, $25 \mathrm{~mm}$ length Nd:YAG single-crystal rod through a $0.64 \mathrm{~m}^{2}$ area Fresnel lens (Liang and Almeida, 2011). This result triggered discussions about which medium between Cr:Nd:YAG ceramics and Nd:YAG single-crystal was more suitable for solar-pumped lasers. Consequently, in 2012, collection efficiency of $30.0 \mathrm{~W} / \mathrm{m}^{2}$ was attained by pumping a $6 \mathrm{~mm}$ diameter, $100 \mathrm{~mm}$ length Nd:YAG single-crystal rod through a $4.0 \mathrm{~m}^{2}$ area Fresnel lens (Dinh et al., 2012). However, very large $\mathrm{M}_{\mathrm{x}}^{2}=\mathrm{M}_{\mathrm{y}}^{2}=137$ factors were associated with this pumping approach, leading to a low laser beam brightness figure of merit - defined as the ratio between laser power and the product of $\mathrm{M}_{\mathrm{x}}{ }^{2}$ and $\mathrm{M}_{\mathrm{y}}{ }^{2}$ (Dinh et al., 2012) - of only $0.0064 \mathrm{~W}$. By end-side-pumping a $4 \mathrm{~mm}$ diameter, $35 \mathrm{~mm}$ length Nd:YAG single-crystal rod with a heliostat-parabolic mirror solar energy concentration system, $31.5 \mathrm{~W} / \mathrm{m}^{2}$ multimode solar laser collection efficiency was reported (Liang et al., 2017). Most recently, a monolithic fused silica liquid light guide lens was used to achieve end-side-pumping of a $4.5 \mathrm{~mm}$ diameter, $35 \mathrm{~mm}$ length Cr:Nd:YAG ceramic laser rod within a conical pumping cavity. $32.5 \mathrm{~W} / \mathrm{m}^{2}$ solar laser collection efficiency and $6.7 \%$ laser slope efficiency were achieved by a parabolic primary mirror. However, large $\mathrm{M}_{\mathrm{x}}^{2}=\mathrm{M}_{\mathrm{y}}^{2}=95$ factors were measured, leading to $0.004 \mathrm{~W}$ laser beam brightness figure of merit (Liang et al., 2018).

Manufacturers view laser material processing as a well-understood productivity enhancement, which they constantly seek to extend to new segments of their business. Lately, that search has produced a trend toward the deployment of multiple laser beams on a single work-

E-mail address: dl@fct.unl.pt (D. Liang) 
piece, each optimized to perform a facet of the overall process (Strite et al., 17th, 2016.). The following laser beam material processing concepts are usually considered.

1. Single-laser/single-beam concept. It is most commonly used in many laser applications, but can be out of any economic value with the current single spot technology due to the enormous processing time of days and even weeks (Bruening et al., 2018).

2. Multi-laser/multi-beam concept. It is based on macroscopic lateral arrangement of many lasers. A further decrease of the processing time is possible by scaling many single lasers. However, due to the size of the lasers and its beam delivery systems, this approach is limited in the maximum number of deployable laser sources.

3. Single-laser/multi-beam concept based on laser beam splitting technologies. This approach has high compactness because one laser serves all spots. However, laser output power and its beam quality is strongly influenced by the thermal lensing and thermal stress problems of the single-laser source.

To avoid the above mentioned limitations, a single-laser/multi-beam concept based on the simultaneous emissions of multi-beam from a single solar laser is here proposed. This approach has also high compactness since one laser serves all spots. Moreover, the above mentioned thermal lensing and thermal stress problems can be significantly reduced. It can deploys three moderate power renewable laser beams, thus providing the capability to tailor the applied solar laser energy to the specific needs of material processing applications. In some space applications (Guan et al., 2017), the concerns on absolute solar laser power level can be relegated to a less important role, while the simultaneous emissions of several solar laser beams at low power level, but with enhanced beam quality, may become more demanding. It is based on these concerns that the three-rod solar laser scheme was designed, implemented and tested. The design parameters of the three-rod within one pump cavity solar laser system, optimized by ZEMAX( $\subseteq$ and LASCAD(C) software, will be explained in Sections 2 and 3. Experiments on continuous-wave three-rod solar laser emissions and three laser beam profiles measurements will then be discussed in Section 4 and 5, finally followed by conclusions in Section 6 .

\section{Three-rod Nd:YAG solar laser system}

\subsection{Solar energy collection and concentration system}

As shown in Fig. 1, NOVA heliostat-parabolic mirror solar laser system was composed of a large plane mirror (composed of two flat segments) with $93.5 \%$ reflectivity, mounted on a two-axis heliostat, which redirected the incoming solar radiation towards a stationary parabolic mirror with $1.5 \mathrm{~m}$ diameter, $60^{\circ}$ rim angle and $660 \mathrm{~mm}$ focal length. The parabolic mirror was back-surface silver coated. Because of

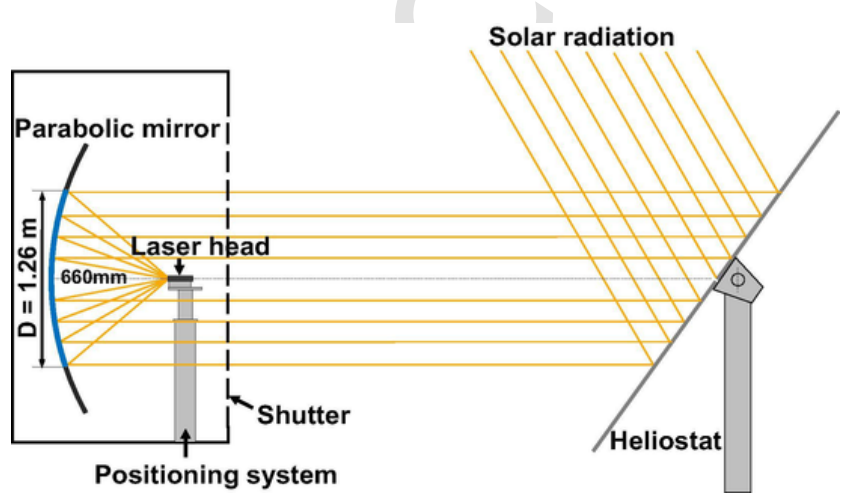

Fig. 1. Schematics of NOVA heliostat - parabolic mirror system for pumping the three-rod solar laser head. iron impurities within $10 \mathrm{~mm}$ glass substrates, $80 \%$ reflectivity was measured for this primary mirror. The total combined reflectance of both the heliostat and the parabolic mirror was $74.8 \%$. The input solar power at the focus was limited by masking the external annular area of the $1.5 \mathrm{~m}$ diameter mirror, so that only its $1.26 \mathrm{~m}$ diameter central circular area was utilized. $1.0 \mathrm{~m}^{2}$ effective solar collection area was calculated by discounting the shadowing area of $0.25 \mathrm{~m}^{2}$ by the laser head, its supporting mechanics, the X-Y-Z positioner and the non-reflecting space between the two flat segments of the heliostat mirrors.

For a typical solar irradiance of $830 \mathrm{~W} / \mathrm{m}^{2}$ in Lisbon, September 2019 , more than $600 \mathrm{~W}$ solar power was focused into a highly concentrated pump light spot with near-Gaussian distribution of $8 \mathrm{~mm}$ full width at half maximum (FWHM).

\subsection{Solar laser head with the aspheric lens, the three Nd:YAG rods and the single pump cavity}

Fig. 2a presents the front image of the solar laser head composed of the large fused silica aspheric lens and the three

$3 \mathrm{~mm}$ diameter, $25 \mathrm{~mm}$ length Nd:YAG rods within the single conical pump cavity. By optically aligning three small partial reflection (PR) $1064 \mathrm{~nm}$ output mirrors with their corresponding laser rods, as shown in Fig. 2b, simultaneous $\mathrm{cw} 1064 \mathrm{~nm}$ solar laser emissions were produced. Accurate resonant cavity alignments were ensured by adjusting the output couplers individually through the three positioners. The laser head was fixed on the X-Y-Z axes positioning system, ensuring its optical alignment in the focal zone.

As shown in Figs. 2 and 3, the large fused silica aspheric lens was $84 \mathrm{~mm}$ in diameter, $34 \mathrm{~mm}$ in thickness, $60 \mathrm{~mm}$ in front surface radius of curvature and -0.003 in rear $\mathrm{r}^{2}$ parameter. The output end face of the lens had a plane surface. The aspheric lens coupled efficiently the concentrated solar radiation from the focal zone into the three Nd:YAG rods. For end-pumping, one part of the concentrated radiation was directly focused onto the high-reflection (HR $1064 \mathrm{~nm}$ ) end face coatings of the rods by the aspheric lens. The HR coatings reflected the $1064 \mathrm{~nm}$ oscillating laser radiation within the resonant cavity, but allowed the passage of other solar pumping wavelengths. For side-pumping, another part of the radiation that did not hit the HR $1064 \mathrm{~nm}$ coatings was guided into the conical cavity with

$\mathrm{D}_{1}=18 \mathrm{~mm} / \mathrm{D}_{2}=9 \mathrm{~mm}$ input/output diameters and $\mathrm{H}_{1}=19 \mathrm{~mm}$ height. The zigzag passage of the rays within the small pump cavity ensured efficient multi-pass side-pumping to the rods. The inner wall of the pumping cavity was bonded with a protected silver-coated aluminum foil with $94 \%$ reflectivity. The Nd:YAG rods, the conical pump cavity and the output end face of the aspheric lens were all actively cooled by water at $6 \mathrm{~L} / \mathrm{min}$ flow rate. The maximum contact between the coolant and the rod was essential for the removal of the generated heat. The central region of the aspheric lens output face was in direct contact with the cooling water, ensuring hence an efficient light coupling of the concentrated solar radiation into the rods. There was $3 \mathrm{~mm}$ space between the aspheric lens output end face and the HR1064 nm coatings of the rods, more than enough for the exit of cooling water. Besides, both fused silica material and cooling water were useful for partially preventing both UV solarization and IR heating to the rods. $\mathrm{L}_{1}, \mathrm{~L}_{2}$ and $\mathrm{L}_{3}$ represent the laser resonant cavity lengths between the laser rods and their respective PR1064 nm mirrors $\left(\mathrm{PR}_{1}, \mathrm{PR}_{2}\right.$ and $\left.\mathrm{PR}_{3}\right)$.

\section{Numerical optimization of the design parameters by ZEMAX(C) and LASCAD@ software}

Similar to our previous numerical analysis on solar lasers (Almeida et al., 2013; Liang and Almeida, 2011), all the above mentioned design parameters of the three-rod solar laser system were firstly optimized by non-sequential ray-tracing ZEMAX@ software for achieving the maximum absorbed pump power for each of the three rods. 

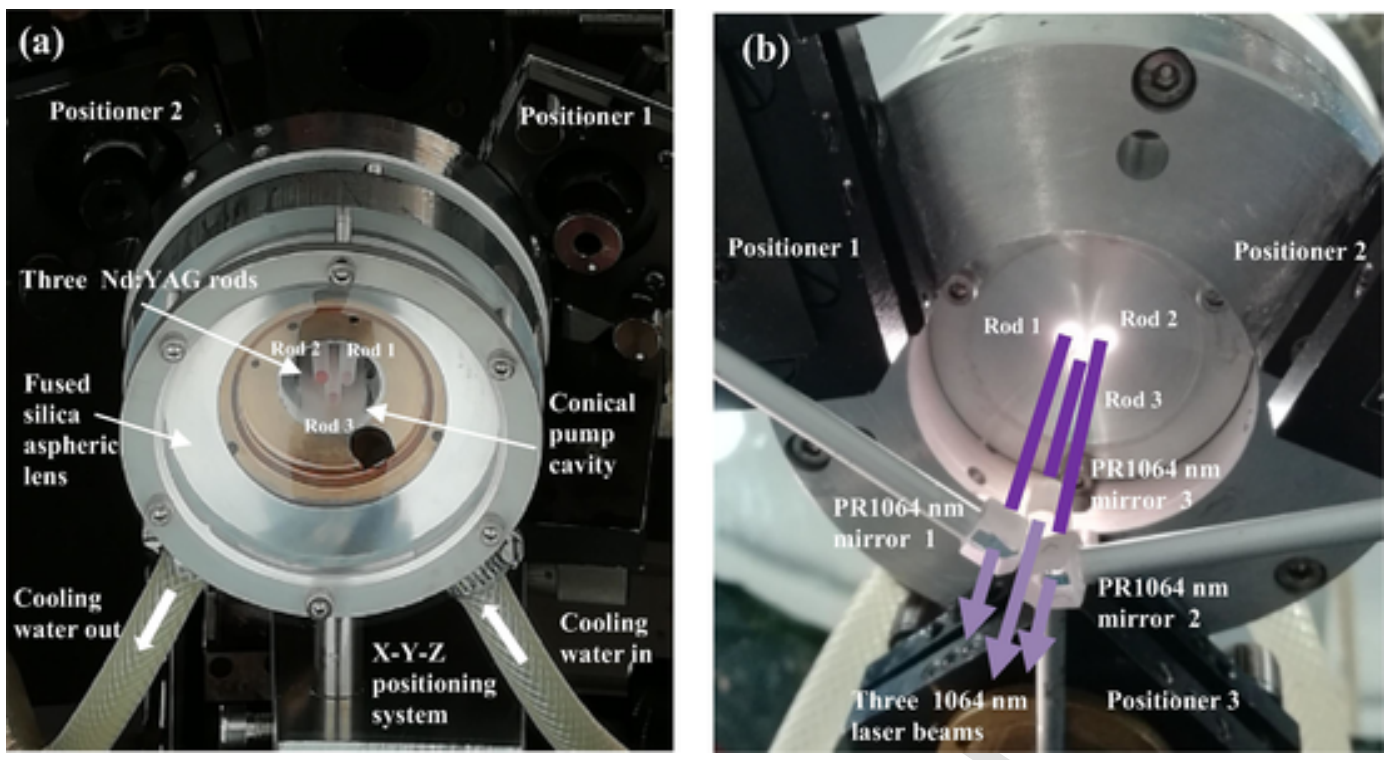

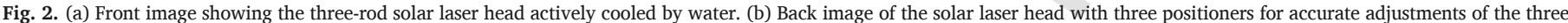
small output couplers in relation to the three rods.

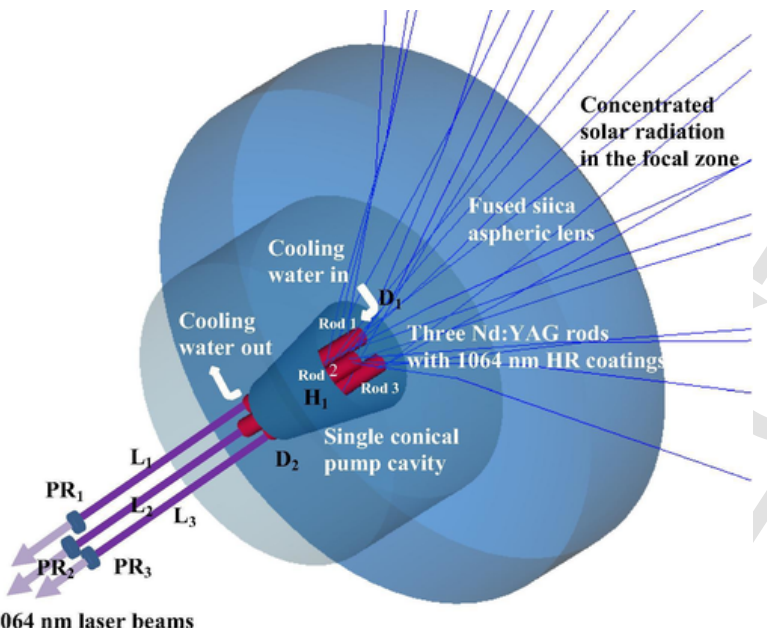

Three $1064 \mathrm{~nm}$ laser beams

Fig. 3. Design of the three-rod Nd:YAG laser head, composed of the fused silica aspheric lens, the conical pump cavity and the Nd:YAG rods, which were all actively cooled by water.

The pump-flux distributions along longitudinal central cross-sections and five transversal cross-sections of the rods are given in Fig. 4. Red color means near maximum pump absorption, whereas blue means little or no absorption.

Non-uniform distributions along the laser rods were numerically calculated. Nevertheless, these were the distributions that ensured the maximum absorbed pump power by the three rods. During ray-tracing, each $3 \mathrm{~mm}$ diameter, $25 \mathrm{~mm}$ length active medium was divided into 18,000 zones. The path length in each zone was found. With this value and the effective absorption coefficient of 1.0 at.\% Nd:YAG material (Liang and Almeida, 2011), the absorbed power within the laser medium was calculated by summing up the absorbed pump radiation of all zones.

The absorbed pump flux data from the ZEMAX(C analysis was then processed by laser cavity analysis and design (LASCADC) software to optimize solar laser output performances. The stimulated emission cross-section of

$2.8 \times 10^{-19} \mathrm{~cm}^{2}$, the fluorescence life time of $230 \mu$ s and a typical absorption and scattering loss of $0.003 \mathrm{~cm}^{-1}$ for the 1.0 at.\%

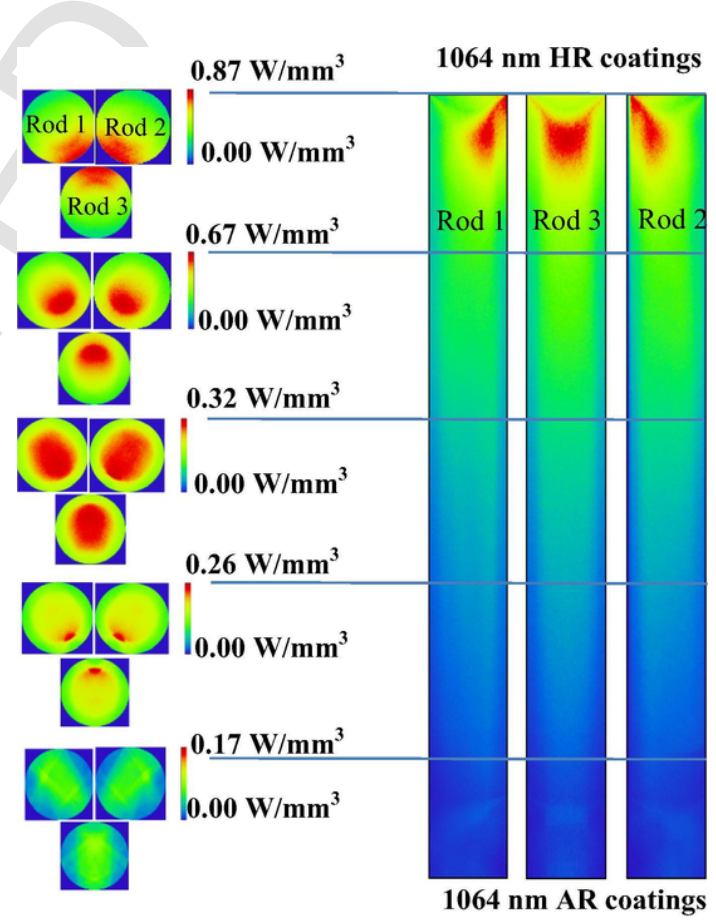

Fig. 4. Absorbed pump-flux distributions along longitudinal central cross-sections and five transversal cross-sections of the three $3 \mathrm{~mm}$ diameter, $25 \mathrm{~mm}$ length Nd:YAG rods.

Nd:YAG medium were adopted in LASCADC analysis. The mean absorbed and intensity-weighted solar pump wavelength of $660 \mathrm{~nm}$ (Weksler and Shwartz, 1988) was also used in the analysis. L represents the separation length between the output end face anti-reflection (AR) $1064 \mathrm{~nm}$ coatings of the Nd:YAG rod and PR1064 nm output mirror, as shown in Fig. 5. L was the key parameter for achieving the highest laser power. PR1064 nm mirrors of different reflectivity (R) and radius of curvature (RoC) were tested individually to optimize laser power for each rod. For the $3 \mathrm{~mm}$ diameter, 1.0 at.\% Nd:YAG rod with length $\mathrm{L}_{\mathrm{R}}=25 \mathrm{~mm}$, the amount of absorption and scattering losses was $2 \alpha \mathrm{L}_{\mathrm{R}}=1.5 \%$. Assuming $0.4 \%$ of imperfect $\mathrm{HR}$ and AR coating losses, the round-trip loss increased to $1.9 \%$. The diffraction losses depend heavily on rod diameter, resonator length and $\mathrm{RoC}$ of the resonator mirrors. LASCADC 

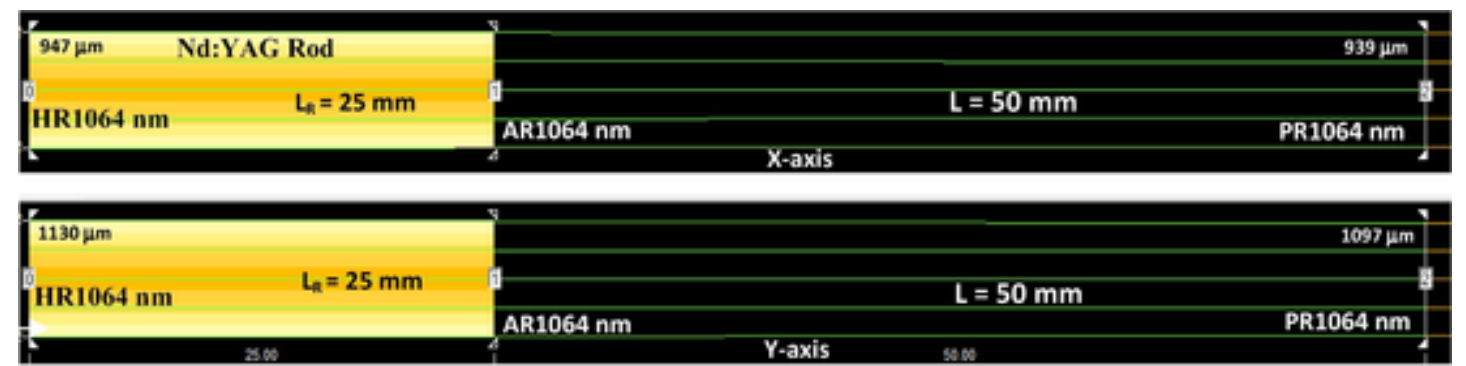

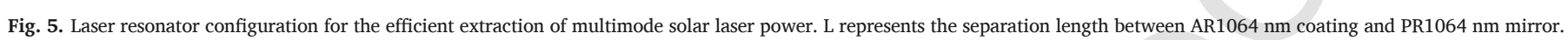

beam propagation method (BPM) gave $0.05 \%$ diffraction loss for $\mathrm{L}=50 \mathrm{~mm}$ and $\mathrm{RoC}=\infty$, resulting in a total round-trip loss of $1.95 \%$. Consequently, $6.2 \mathrm{~W}$ multimode solar laser power was numerically obtained. The half-widths of the oscillating modes within the resonant cavity (947 $\mu \mathrm{m}$ in X-axis, $1130 \mu \mathrm{m}$ in Y-axis at HR1064 $\mathrm{nm}$ mirror) and (939 $\mu \mathrm{m}$ in X-axis, $1097 \mu \mathrm{m}$ in Y-axis at PR1064 $\mathrm{nm}$ mirror) were also calculated, as shown in Fig. 5. The laser beam quality $\mathrm{M}_{\mathrm{x}}{ }^{2}=10.8$, $\mathrm{M}_{\mathrm{y}}{ }^{2}=14.7$ factors were then numerically obtained for the PR1064 nm mirror RoC $=\infty$.

In a single-rod solar laser pumping scheme, instead of adopting three $3 \mathrm{~mm}$ diameter, $25 \mathrm{~mm}$ length Nd:YAG rods within the conical pump cavity in Fig. 3, a $5.2 \mathrm{~mm}$ diameter, $25 \mathrm{~mm}$ length $\mathrm{Nd}$ :YAG rod could also be pumped within the same conical cavity under the same solar pumping conditions as explained in Section 2.2. Since the volume of the $5.2 \mathrm{~mm}$ diamer $25 \mathrm{~mm}$ length rod was nearly the same as the sum of the volumes of the three $3 \mathrm{~mm}$ diameter, $25 \mathrm{~mm}$ length rods, the solar laser output performances of both the single-rod and the three-rod solar laser pumping schemes were also analyzed by both ZEMAX(C and LASCADC software, as listed in Table 1.

Due to the gaps between the rods, the three-rod solar laser pumping scheme absorbed $4.7 \%$ less solar pump power, and consequently $11 \%$ less solar laser power was numerically calculated, as compared to that by the single-rod scheme. Because of the assymmetric and inhomogenious absorbed pump light distribution along each rod in Fig. 4, the three-rod scheme presented some discrepences between $\mathrm{M}_{\mathrm{x}}{ }^{2}$ and $\mathrm{M}_{\mathrm{y}}{ }^{2}$ factors. The $\mathrm{M}^{2}$ factors of each laser beam from the three-rod scheme were, however, 2.97 (for $\mathrm{M}_{\mathrm{x}}{ }^{2}$ ), 2.18 (for $\mathrm{M}_{\mathrm{y}}{ }^{2}$ ) times smaller than that from the single-rod scheme. Consequently, the laser beam brightness figure of merit for each beam was nearly doubled as compared to that of the single-rod scheme. Besides, the three-rod scheme presented $31.6 \%$ reduction in the maximum heat load value as compared to that of the single-rod scheme. It also presented only $303 \mathrm{~K}$ maximum temperature value for each rod, which was $22 \mathrm{~K}$ less than that of the single-rod Scheme $16.9 \mathrm{~N} / \mathrm{mm}^{2}$ maximum thermal stress value was calculated for the three-rod scheme, corresponding to $65.5 \%$ reduction as compared to that of the single-rod scheme.

\section{Simultaneous continuous-wave solar laser emissions from the three Nd:YAG rods}

Based on the ZEMAX@ and LASCAD( numerically optimized design parameters of the three-rod solar laser system in Section 3, a solar laser prototype was firstly designed and built in Lisbon in the first half of 2019, and then tested by both the PROMES-CNRS and the NOVA solar facilities during July - September 2019. Continuous-wave $1064 \mathrm{~nm}$ solar laser beams were successfully obtained from the three rods in both facilities, gaining more solar-to-laser conversion efficiency and accomplishing laser beam profiles measurements by the NOVA facility in the last weeks of September 2019.

As shown in Figs. 4 and 5, each $3 \mathrm{~mm}$ diameter, $25 \mathrm{~mm}$ length Nd:YAG rod was HR1064 nm coated

( $\mathrm{R} \geq 99.8 \% @ 1064 \mathrm{~nm}$ ) on one end face and AR1064 nm coated ( $\mathrm{R} \leq 0.2 \% @ 1064 \mathrm{~nm}$ ) on the other end face. To achieve maximum solar laser output power with enhanced beam brightness, each $\mathrm{R}=95 \%$,

$\mathrm{RoC}=\infty$ PR1064 $\mathrm{nm}$ mirror was mounted $50 \mathrm{~mm}$ away from the AR $1064 \mathrm{~nm}$ output face of each laser rod, as indicated in Fig. 3. By varying the rotation angle of the shutter, different input solar power and output laser power were respectively measured with a Molectron PowerMax 500D and a Thorlabs PM1100D power meters.

Table 1

Numerically calculated laser output performances of both the single-rod and the three-rod pumping schemes.

\begin{tabular}{|c|c|c|c|c|c|c|c|}
\hline & $\begin{array}{l}\text { Solar pump power } \\
\text { absorption efficiency }\end{array}$ & $\begin{array}{l}\text { Multimode solar } \\
\text { laser power }\end{array}$ & $\begin{array}{l}\text { Laser beam } \\
\mathrm{M}^{2} \text { factors }\end{array}$ & $\begin{array}{l}\text { Laser beam brightness } \\
\text { figure of merit }\end{array}$ & Heat load & Temperature & $\begin{array}{l}\text { Thermal } \\
\text { stress }\end{array}$ \\
\hline $\begin{array}{l}5.2 \mathrm{~mm} \text { diameter, } 25 \mathrm{~mm} \text { length } \\
\text { rod } \\
\text { Single-rod scheme }\end{array}$ & $44.8 \%$ & $20.9 \mathrm{~W}$ & $\begin{array}{l}\mathrm{M}_{\mathrm{x}}^{2}=32.1 \\
\mathrm{M}_{\mathrm{y}}^{2}=32.1\end{array}$ & $0.02 \mathrm{~W}$ & & & \\
\hline $\begin{array}{l}3.0 \mathrm{~mm} \text { diameter } 25 \mathrm{~mm} \text { length } \\
\text { rods } \\
\text { Three-rod scheme }\end{array}$ & $40.1 \%$ & $\begin{array}{l}6.2 \mathrm{~W} \\
\text { for each rod } \\
3 \times 6.2 \mathrm{~W} \\
=18.6 \mathrm{~W} \text { for } \\
\text { three rods }\end{array}$ & $\begin{array}{l}\mathrm{M}_{\mathrm{x}}^{2}=10.8 \\
\mathrm{M}_{\mathrm{y}}^{2}=14.7\end{array}$ & $0.039 \mathrm{~W}$ & & & \\
\hline $\begin{array}{l}\text { Compari-son between three-rod } \\
\text { and single-rod schemes }\end{array}$ & $4.7 \%$ reduction & $11 \%$ reduction & $\begin{array}{l}2.97 \text { times } \\
\text { reduction for } \\
\mathrm{M}_{\mathrm{x}}^{2} \\
2.18 \text { times } \\
\text { reduction for } \\
\mathrm{M}_{\mathrm{y}}{ }^{2}\end{array}$ & $\begin{array}{l}1.95 \text { times } \\
\text { Improve-ment }\end{array}$ & $\begin{array}{l}31.6 \% \\
\text { reduction }\end{array}$ & $\begin{array}{l}22 \mathrm{~K} \\
\text { reduction }\end{array}$ & $\begin{array}{l}65.5 \% \\
\text { reduction }\end{array}$ \\
\hline
\end{tabular}


Direct solar irradiance was measured simultaneously during the lasing process. It varied between $800 \mathrm{~W} / \mathrm{m}^{2}$ and

$840 \mathrm{~W} / \mathrm{m}^{2}$. After considering all the shading effects in the primary concentrator, an effective collection area of $1.0 \mathrm{~m}^{2}$ was adopted. For $830 \mathrm{~W} / \mathrm{m}^{2}$ solar irradiance, $620 \mathrm{~W}$ solar power was measured at the focus of the primary concentrator. As shown in Fig. 6, maximum multimode solar laser power of $18.3 \mathrm{~W}$ was successfully registered, corresponding to $18.3 \mathrm{~W} / \mathrm{m}^{2}$ collection efficiency. $6.1 \mathrm{~W}$ average solar laser power from each rod was hence obtained. Threshold solar pump power of $500 \mathrm{~W}$ was also measured, resulting in the solar laser slope efficiency of $5.1 \%$.

5. Solar laser beam profile and its dependency on resonant cavity length and output coupler optical alignment

The laser beam $\mathrm{M}^{2}$ quality factor of each laser rod at $\mathrm{L}=50 \mathrm{~mm}$ was measured according to ISO 11146-1 standards, by using a CINOGY UV-NIR beam profiler - CinCam CMOS. The measured solar laser beam widths along the beam caustic, together with the extrapolated hyperbolic plot of the measured data, are given in Fig. 7. For the RoC $=\infty$ PR1064 nm mirror, $\mathrm{M}_{\mathrm{x}}{ }^{2}=11.0, \mathrm{M}_{\mathrm{y}}{ }^{2}=15.3$ were experimentally determined for one laser rod, leading to the beam brightness figure of merit of $0.036 \mathrm{~W}$, being 9 times higher than that of the most efficient solar lasers with $32.5 \mathrm{~W} / \mathrm{m}^{2}$ collection efficiency (Liang et al., 2018).

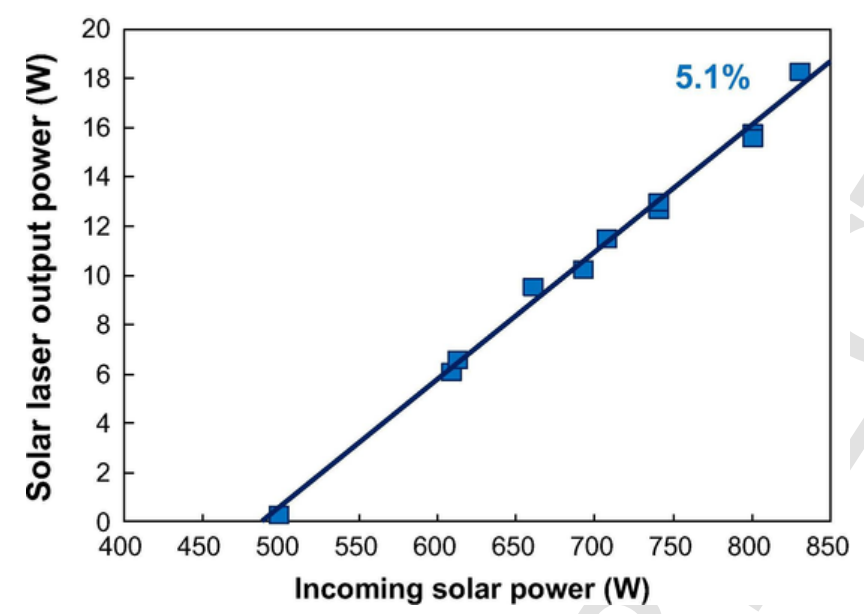

Fig. 6. Total solar laser output power from the three rods versus incoming solar power for $\mathrm{R}=95 \%$, RoC $=\infty$ and $\mathrm{L}_{1}=\mathrm{L}_{2}=\mathrm{L}_{3}=50 \mathrm{~mm}$.

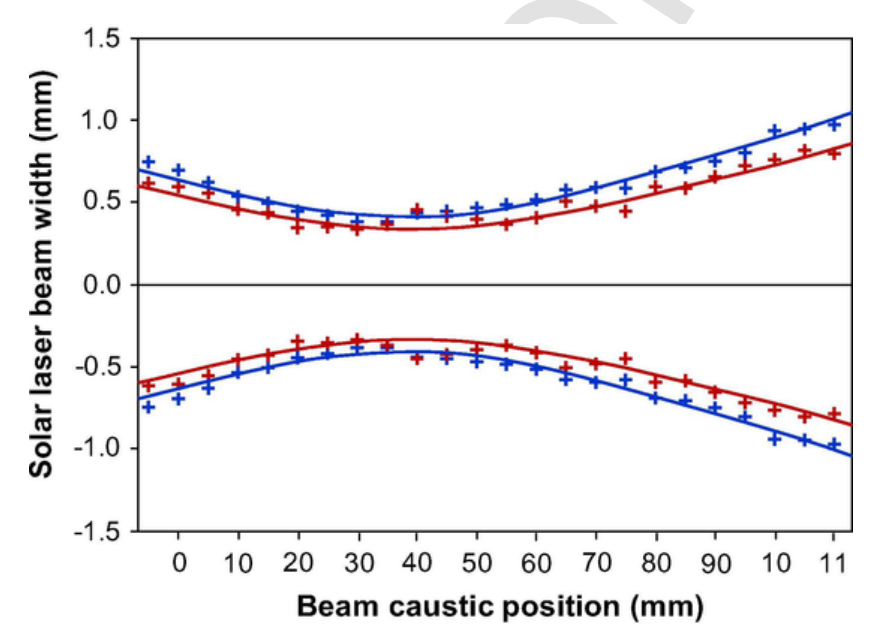

Fig. 7. Caustic fit measurements of the multimode solar laser beam from one of the three rods.
Different laser output beam profiles from the three laser rods were achieved by adjusting the PR mirrors of the solar laser resonant cavity. Fig. 8a indicates the output beam profiles with one sharp peak and two weak peaks when there was accurate laser resonant cavity alignment of the PR1064 nm mirror 3, while PR1064 nm mirror 1 and 2 were poorly aligned. $8.9 \mathrm{~W}$ total solar laser power was measured in this case. Fig. 8b shows different laser beam profiles with two strong peaks and one weak peak when the

PR1064 nm mirror 2 and 3 were well aligned and the PR1064 nm mirror 1 was not accurately aligned.

12.3 $\mathrm{W}$ total solar laser power was measured. When all the three PR1064 nm mirrors were accurately aligned with their respective laser rods, solar laser beam profiles with three sharp peaks were achieved, as shown in Fig. 8c. The maximum total solar laser power of $18.3 \mathrm{~W}$ was measured.

By choosing different laser resonant cavity lengths for the three rods, significantly different output laser beam profiles were achieved. As shown in Fig. 8d, a sharp peak from the rod 1 was observed with the resonator cavity length $\mathrm{L}_{1}=65 \mathrm{~mm}$, while other two relatively weak peaks were observed from the rod 2 and 3 by adopting $\mathrm{L}_{2}=48 \mathrm{~mm}$ and $\mathrm{L}_{3}=56 \mathrm{~mm}$, respectively. $15.4 \mathrm{~W}$ total solar laser power was measured. The simultaneous production of three laser beams with considerably different profiles may constitute an advantage for the three-rod solar laser scheme in finding more applications. Enhanced solar laser power could be achieved if PR1064 nm mirrors with small RoC were adopted. However, large $\mathrm{M}^{2}$ factors would be measured, which inevitably would result in laser beams with reduced brightness figure of merit.

\section{Conclusions}

The three-rod Nd:YAG solar laser scheme was composed of the first-stage heliostat-parabolic mirror solar energy collection and concentration system, the second-stage fused silica aspheric lens and the third-stage conical-shaped pumping cavity, within which the three $3 \mathrm{~mm}$ diameter, $25 \mathrm{~mm}$ length Nd:YAG rods were efficiently pumped. Optimum optical pumping system design parameters were found through ZEMAX( software. Optimum solar laser power and beam parameters were found through LASCADC numerical analysis. $18.3 \mathrm{~W}$ continuous-wave multimode total solar laser power was measured, corresponding to $5.1 \%$ system slope efficiency and $18.3 \mathrm{~W} / \mathrm{m}^{2}$ solar laser collection efficiency. $0.036 \mathrm{~W}$ laser beam brightness figure of merit was also obtained, being 9 times higher than that of the most efficient solar laser with $32.5 \mathrm{~W} / \mathrm{m}^{2}$ collection efficiency (Liang et al., 2018). By increasing the resonant cavity length from the AR1064nm laser rod end face, different solar laser output power and beam profiles were obtained for the three rods. Simultaneous solar laser emissions from the three $3 \mathrm{~mm}$ diameter rods could provide new solutions for solar-powered lasers applications.

\section{Funding information}

Financial support of the strategic project (UID/FIS/00068/2019) of the Science and Technology Foundation of Portuguese Ministry of Science, Technology and Higher Education (FCT - MCTES) is acknowledged. Financial support by the Solar Facilities for European Research Area -Third Phase (SFERA III), Grant Agreement No. 823802 is gratefully acknowledged.

\section{Acknowledgment}

FCT-MCTES grants SFRH/BPD/125116/2016, PD/BD/142827/ 2018, PD/BD/128267/2016 of C. R.Vistas, D. Garcia, B. D. Tibúrcio, respectively, and FCT-MCTES Junior Researcher Contract CEECIND/ 03081/2017 of J. Almeida are acknowledged. 
(a)

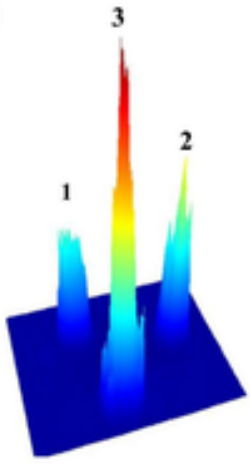

Accurate alignment of the PR1064 nm mirror 3

(c)
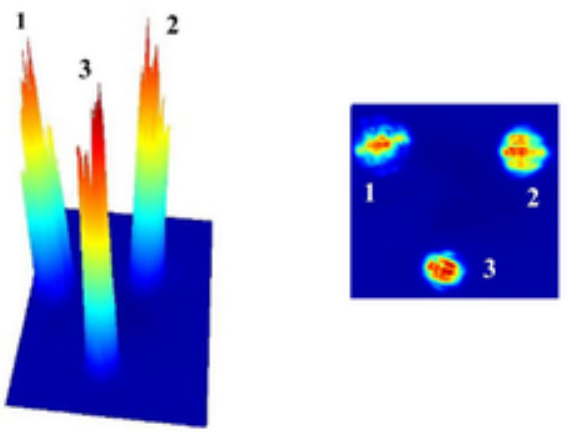

Accurate alignments of the PR1064 nm mimor 1, 2 and 3 (b)

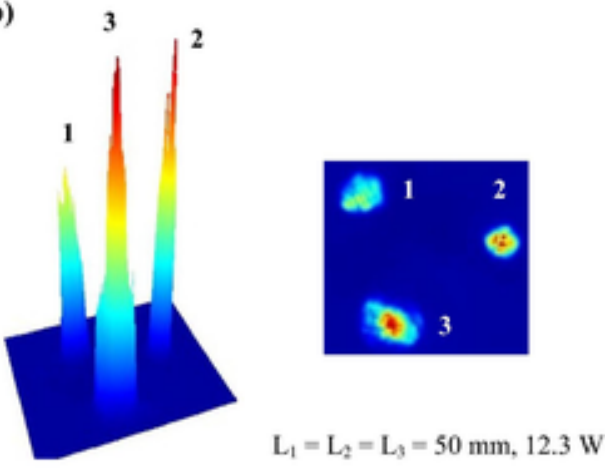

Accurate alignments of the PR1064 nm mimors 2 and 3

(d)

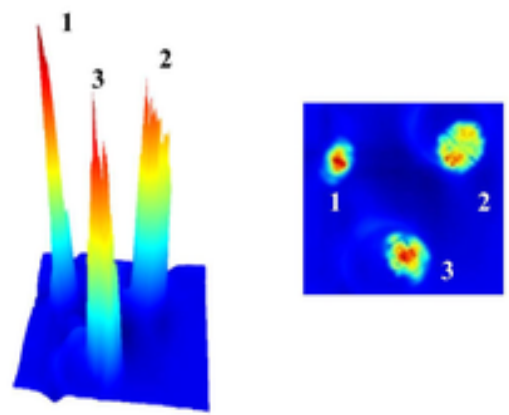

$\mathrm{L}_{1}=65 \mathrm{~mm}, \mathrm{~L}_{2}=48 \mathrm{~mm}, \mathrm{~L}_{3}=56 \mathrm{~mm}, 15.4 \mathrm{~W}$

Accurate alignments of the PR1064 nm mimors 1,2 and 3

Fig. 8. Measured output laser beam 3D and 2D profiles of the three-rod laser system at different resonant cavity alignments and lengths.

\section{References}

Almeida, J., Liang, D., Guillot, E., Abdel-Hadi, Y., 2013. A 40 W cw Nd:YAG solar laser pumped through a heliostat: a parabolic mirror system. Laser Phys. 23. 065801 065801.

Arashi, H., Oka, Y., Sasahara, N., Kaimai, A., Ishigame, M., 1984. A solar-pumped cw 18 W Nd:YAG laser. Jpn. J. Appl. Phys. 23, 1051-1053.

Benmair, R.M.J., Kagan, J., Kalisky, Y., Noter, Y., Oron, M., Shimony, Y., Yogev, A., 1990. Solar-pumped Er, Tm, Ho: YAG laser. Opt. Lett. 15, 36-38.

Bruening, S., Du, K., Jarczynski, M., Jenke, G., Gillner, A., 2018. Ultra-fast laser micro processing by multiple laser spots. Procedia CIRP 74, 573-580.

Dinh, T.H., Ohkubo, T., Yabe, T., Kuboyama, H., 2012. 120 watt continuous wave solar-pumped laser with a liquid light-guide lens and a Nd:YAG rod. Opt. Lett. 37, 2670-2672.

Guan, Z., Zhao, C., Yang, S., Wang, Y., Ke, J.Y., Zhang, H., 2017. Demonstration of a free-space optical communication system using a solar-pumped laser as signal transmitter. Laser Phys. Lett. 14 (5), 55804.

Lando, M., Kagan, J., Linyekin, B., Dobrusin, V., 2003. A solar pumped Nd:YAG laser in the high collection efficiency regime. Opt. Commun. 222, 371-381.
Liang, D., Almeida, J., 2011. Highly efficient solar-pumped Nd:YAG laser. Opt. Exp. 19, 26399-26405.

Liang, D., Almeida, J., Vistas, C.R., Guillot, E., 2017. Solar-pumped Nd:YAG laser with $31.5 \mathrm{~W} / \mathrm{m}^{2}$ multimode and $7.9 \mathrm{~W} / \mathrm{m}^{2} \mathrm{TEM}_{00}$-mode collection efficiencies. Sol. Energy Mat. Sol. Cells 159, 435-439.

Liang, D., Vistas, C.R., Tíbúrcio, B.D., Almeida, J., 2018. Solar-pumped Cr:Nd:YAG ceramic laser with 6.7\% slope efficiency. Sol. Energy Mat. Sol. Cells 185, 75-79.

Strite, T., Gusenko, A., Grupp, M., Hoult, T., 17th, 2016.. Fiber lasers: Multiple laser beam material processing. Laser Focus World, Feb..

Vasile, M., Maddock, C.A., 2012. Design of a formation of solar pumped lasers for asteroid deflection. Adv. Space Res. 50 (7), 891-905

Weksler, M., Shwartz, J., 1988. Solar-pumped solid-state lasers. IEEE J. Quantum Electron. $24,1222-1228$

Yabe, T., Ohkubo, T., Uchida, S., Nakatsuka, M., Funatsu, T., Mabuti, A., Oyama, A., Nakagawa, Y., Oishi, T., Daito, K., Behgol, B., Nakayama, Y., Yoshida, M., Motokoshi, S. Sato, Y., Baasandash, C., 2007. High efficiency and economical solar energy pumped laser with Fresnel lens and chromium co-doped laser medium. Appl. Phys Lett. 90, $261120-261123$

Young, C.G., 1966. A sun pumped cw one-watt laser. Appl. Opt. 5, 993-997. 